\title{
The spectrum of psychosis in multiple sclerosis: a clinical case series
}

\author{
This article was published in the following Dove Press journal: \\ Neuropsychiatric Disease and Treatment \\ I February 2017 \\ Number of times this article has been viewed
}

\author{
Thomas G Gilberthorpe' \\ Kara E O'Connell ${ }^{2,3}$ \\ Alison Carolan' \\ Eli Silber ${ }^{4}$ \\ Peter A Brex ${ }^{4}$ \\ Naomi A Sibtain ${ }^{4}$ \\ Anthony S David ${ }^{3}$ \\ 'Department of Liaison Psychiatry, \\ South London and Maudsley \\ NHS Foundation Trust, Maudsley \\ Hospital, London, UK; ${ }^{2}$ Department \\ of Psychiatry, School of Medicine, \\ Trinity College Dublin, Ireland; \\ ${ }^{3}$ Department of Psychosis Studies, \\ Institute of Psychiatry, Psychology \\ and Neuroscience, ${ }^{4}$ Department of \\ Neurology, Kings College Hospital, \\ London, UK
}

Correspondence: Thomas G Gilberthorpe South London and Maudsley NHS Foundation Trust, Maudsley Hospital, Denmark Hill, London SE5 8AZ, UK Tel +442032286000

Email thomas.gilberthorpe@slam.nhs.uk

\begin{abstract}
Psychosis in the context of multiple sclerosis (MS) has previously been reported as a rare occurrence. However, recent epidemiological studies have found prevalence rates of psychosis in MS that are two to three times higher than those in the general population. Untreated psychosis in patients with MS can adversely impact on adherence to MS medication, levels of disability, and quality of life. This retrospective case series describes the spectrum of psychotic disorders occurring in association with MS using demographic, clinical, and neuroimaging data. In the discussion, we highlight the particular diagnostic and treatment challenges that such disorders can pose for clinicians and through our case vignettes provide examples of potential interventions for this complex patient population.
\end{abstract}

Keywords: psychosis, multiple sclerosis, neuropsychiatric, cognitive, schizophrenia-like, demyelination

\section{Introduction}

Multiple sclerosis (MS) is a chronic inflammatory disease of the central nervous system (CNS) characterized by progressive demyelination. It can affect any part of the CNS but has a predilection for the white matter tracts of the cerebral hemispheres, optic nerves, brainstem, cerebellum, and spinal cord. ${ }^{1,2}$

It is the most common cause of neurological disability in young and middle-aged adults, with recent UK prevalence estimates of 167 in 100,000 and an annual incidence of eight in $100,000 .^{3,4}$ It is two to three times more common in females than in males and has an average age of onset of $\sim 30$ years. ${ }^{5}$ Typically, MS presents with motor and sensory symptoms such as limb weakness, visual disturbance, ataxia, bladder and bowel dysfunction, and sensory deficits. The majority ( $85 \%$ ) of cases of MS have a relapsing-remitting (RR) course, two-thirds of which go on to develop a secondary progressive (SP) form, and one-third continuing with an RR course that does not result in severe disability. The remaining $15 \%$ of patients have a primary progressive (PP) course with gradual worsening of symptoms over time. ${ }^{6}$ Neuropsychiatric symptoms including mood, cognitive, and behavioral abnormalities occur commonly in MS. ${ }^{7-9}$ One study using the neuropsychiatric inventory found that up to $95 \%$ of MS patients had some form of neuropsychiatric symptoms even at an early stage or mild severity of disease. Depression and dysphoria were found most frequently, occurring in $\sim 80 \%$ of cases; anxiety, agitation, and irritability were found in approximately one-third (33\%); and euphoria, disinhibition, and psychotic symptoms were found in a significantly smaller number of patients $(<13 \%){ }^{10}$

Cognitive impairment is common in MS and is said to affect between $30 \%$ and $70 \%$ of patients at some point during the course of the disease. ${ }^{11}$ A large study by Borghi et $\mathrm{al}^{12}$ 
investigated the presence and significant determinants of cognitive impairment in MS patients versus healthy controls using the Brief Repeatable Battery (BRB) and found rates of $35.6 \%$, where "cognitive impairment" was defined as having two or more BRB test scores of at least 1.5 standard deviations below the scores of healthy controls. A progressive course of MS, older age, the presence of depression, and less time in education were found to be predictors of a more severe cognitive profile in MS patients. ${ }^{12} \mathrm{~A}$ further study by Borghi et $\mathrm{al}^{13}$ followed up patients and healthy controls over a 2-year period and found that cognitive abilities typically compromised in the early stages of MS (attention, working memory, speed of information processing, and verbal fluency) are also more vulnerable to progression compared with healthy controls, suggesting a need to identify effective rehabilitation treatments that might counteract this decline over time. Previous literature shows that patients with a psychiatric disorder and MS tend to have a more severe course of their MS than those without psychiatric morbidity. ${ }^{14,15}$ Such neuropsychiatric disorders have been associated with decreased adherence to MS treatment and poorer outcomes in terms of social and occupational functioning and quality of life. ${ }^{16-18}$ A recent study by Berrigan et al ${ }^{19}$ looked at direct and indirect effects of comorbidity on health-related quality of life (HRQoL) in a large sample of MS patients $(n=949)$ and found that after disability status, depression, and, to a lesser extent, anxiety were associated with decreased HRQoL. A study by Ostacoli et $\mathrm{al}^{20}$ looked at the prevalence and determinants of posttraumatic stress disorder (PTSD) in a large sample of patients with MS. Twelve of 232 (5.17\%) patients fulfilled the diagnostic criteria for PTSD, and two main determinants of PTSD were found to be statistically significant, high anxiety levels and low education level. Both factors are likely to be important in planning psychological interventions to improve levels of functioning and quality of life for patients with MS. ${ }^{20}$

Psychotic features in the context of MS occur rarely but are far from insignificant, with reported prevalence rates two to three times higher than those found in the general population. ${ }^{21}$ A large epidemiological study by Patten et $\mathrm{al}^{22}$ involving $>10,000 \mathrm{MS}$ patients in Canada found that between $2 \%$ and $4 \%$ had experienced psychotic symptoms and met the criteria for a diagnosis of psychotic disorder (including organic and nonorganic psychosis). An association between MS and psychosis has also been suggested by studies of psychiatric inpatients where the prevalence of MS was found to be elevated. Pine et $\mathrm{al}^{23}$ identified 10 patients with MS out of a total of 2,720 psychiatric inpatients (five of whom had psychotic illnesses). Another study found that 23 out of 2,783 psychiatric inpatients had white matter lesions on magnetic resonance imaging (MRI) suggestive of MS (four of these patients had a diagnosis of schizophrenia). ${ }^{24}$

There are rare case reports of neuropsychiatric symptoms, particularly cognitive deficits and to a lesser degree psychotic phenomena, being the presenting manifestation of MS. Assouad et $\mathrm{al}^{25}$ presented 18 cases of "cognitive MS" where 1) cognitive symptoms were the primary and predominant manifestation at disease onset, 2) defined criteria for MS diagnosis were met, and 3) alternative causes were excluded. A small number of case reports document MS presenting as psychosis in the absence of hard neurological signs. ${ }^{26-28}$

Both affective and schizophrenia-like psychotic syndromes have been reported in MS with a predominance of positive psychotic symptoms, most frequently persecutory delusions, and a relative absence of negative symptoms (as seen in schizophrenia). ${ }^{29-31}$ Feinstein et $\mathrm{al}^{14}$ compared the clinical and MRI characteristics of 10 psychotic patients with MS against 10 nonpsychotic controls and found that compared to schizophrenia, MS-related psychosis presents later (mean age 36 years), there is quicker resolution of symptoms (a mean of 5 weeks), fewer relapses $(60 \%$ had only one episode), and better long-term outcomes $(90 \%$ remission). The main neuroimaging finding in this study was a higher periventricular lesion load (particularly around the temporal horns of the lateral ventricles) in psychotic patients. ${ }^{14}$ A number of other MRI studies offer support for an association between temporal lobe, as well as frontotemporal and temporoparietal lesions, and psychosis in MS. ${ }^{21,28,32}$

The exact etiology of psychosis in MS is not known. However, the following three postulations have emerged: 1) psychosis and MS are not separate comorbid disorders but are thought to share a common pathophysiological process (infectious or immunological); ${ }^{21} 2$ ) psychotic symptoms occur as a result of regional demyelination; ${ }^{14,32}$ and 3) psychotic symptoms are caused or exacerbated by medications used to treat MS, for example, corticosteroids and beta interferon. ${ }^{33-36}$

The broad purpose of this study is to describe the spectrum of psychotic disorders found in association with MS. In particular, we are interested in describing the phenomenology of such psychosis and exploring the possibility that psychosis can be the sole presenting feature of MS. Our secondary aims are to further explore the risk factors for psychosis in patients with MS and to review the psychopharmacological management of such patients. 


\section{Methods}

This study is a retrospective case series of patients with a diagnosis of psychotic disorder and either 1) a definitive diagnosis of MS or 2) MRI evidence of demyelination in the absence of neurological symptoms (known as a radiologically isolated syndrome [RIS]). Ethical approval for the study was not sought in accordance with the Kings College Hospital and Maudsley Hospital ethics and clinical governance rules which deem that historical case files may be used in an anonymized fashion in a clinical report without specific consent being obtained.

A total of 15 patients were included in this study having been referred to the Neuropsychiatry Service at Kings College Hospital and/or the Maudsley Hospital within the 5-year period between 2010 and 2015. The main referral source was Kings College Hospital Multiple Sclerosis Service with a small number of patients referred from general psychiatric services seeking a specialist neuropsychiatric opinion.

Of this total, 13 patients had a diagnosis of MS confirmed by a neurologist with a special interest in MS using revised McDonald criteria. ${ }^{37,38}$ The remaining two patients had MRI scans suggestive of demyelination but no hard neurological findings on review by a neurologist. Hence, they did not meet criteria for a diagnosis of MS and were identified as having RISs.

All patients had an International Classification of Diseases, 10th edition (ICD-10), diagnosis of psychotic disorder, made by a psychiatrist during the study period. The term psychotic disorder stipulated the presence of delusions and/or hallucinations in the absence of delirium, allowing schizophrenia, schizophrenia-like, and affective psychosis to be included. Patients with mood disturbance or cognitive impairment without evidence of psychosis were excluded. The temporal relationship between MS onset and onset of first psychotic symptoms was deliberately left open so that the bidirectional relationship between the two disorders could be explored.

Cognitive impairment was rated as mild, moderate, or severe based on cognitive assessment scores. In two-thirds of cases, bedside cognitive tests such as the Mini-Mental State Examination and the Addenbrooke's Cognitive Examination were used. The remaining third (all of whom were inpatients on the neuropsychiatric unit) underwent more detailed neuropsychological assessment using a battery of tests covering the main cognitive domains as follows:

- General intellectual ability using the Wechsler Adult Intelligence Scale-Fourth Edition and Test of Premorbid Functioning;

- Memory using the Brain Injury Rehabilitation Trust Memory and Information Processing Battery;
- Language using the Graded Naming Test, the Letter Fluency Test, and the Category Fluency Test, and

- Executive function using the Delis-Kaplan Executive Function System.

Clinical, demographic, and neuroimaging (MRI) data were collected retrospectively from medical and psychiatric electronic notes. Demographic data consisted of gender, age, age at onset of MS and age at onset of first psychotic symptoms. Clinical data included type of MS (PP, SP, or RR), neurological symptoms/level of disability, degree of cognitive impairment, medical comorbidities, psychotic phenomenology, ICD-10 psychiatric diagnosis, past psychiatric history, risk factors for psychosis such as family history and illicit drug use and antipsychotic treatment. MRI data consisted of the main findings as reported by a neuroradiologist. The reports were then reviewed for comments relating to 1 ) evidence of dissemination of lesions in time and/or space and 2) evidence of lesions in periventricular regions and temporal lobes (as previously associated with psychotic symptoms in MS and other neurological disorders). ${ }^{14,28,32}$

\section{Results}

The sample consisted of 15 cases identified over 5 years with psychosis and MS or an RIS, which is in keeping with previously reported low incidence rates of psychosis associated with MS (Tables 1 and 2).

Table 1 gives an overview of all demographic and clinical characteristics for the 15 cases. Table 2 shows the main MRI findings, psychotic features, and psychopharmacological treatment for each patient.

\section{Demographic and clinical characteristics}

The study sample comprised nine males and six females. Of these, 13 patients had a confirmed MS diagnosis and two had RISs.

The median age at MS diagnosis in the 13 confirmed cases was 33 years (range, 17-50 years). Ten patients had a progressive form of MS and three had RR disease. In keeping with this finding, significant neurological disability was observed in nearly half of the sample, with seven patients being wheelchair bound with a severe degree of bladder and bowel dysfunction. Eleven patients had evidence of cognitive impairment that was assessed as either mild (six cases) or moderate (five cases) based on neuropsychological assessment. Eight cases had medical comorbidities, six of which had an autoimmune or inflammatory basis including diabetes mellitus, hypothyroidism, and asthma. None of the cases had a positive family 


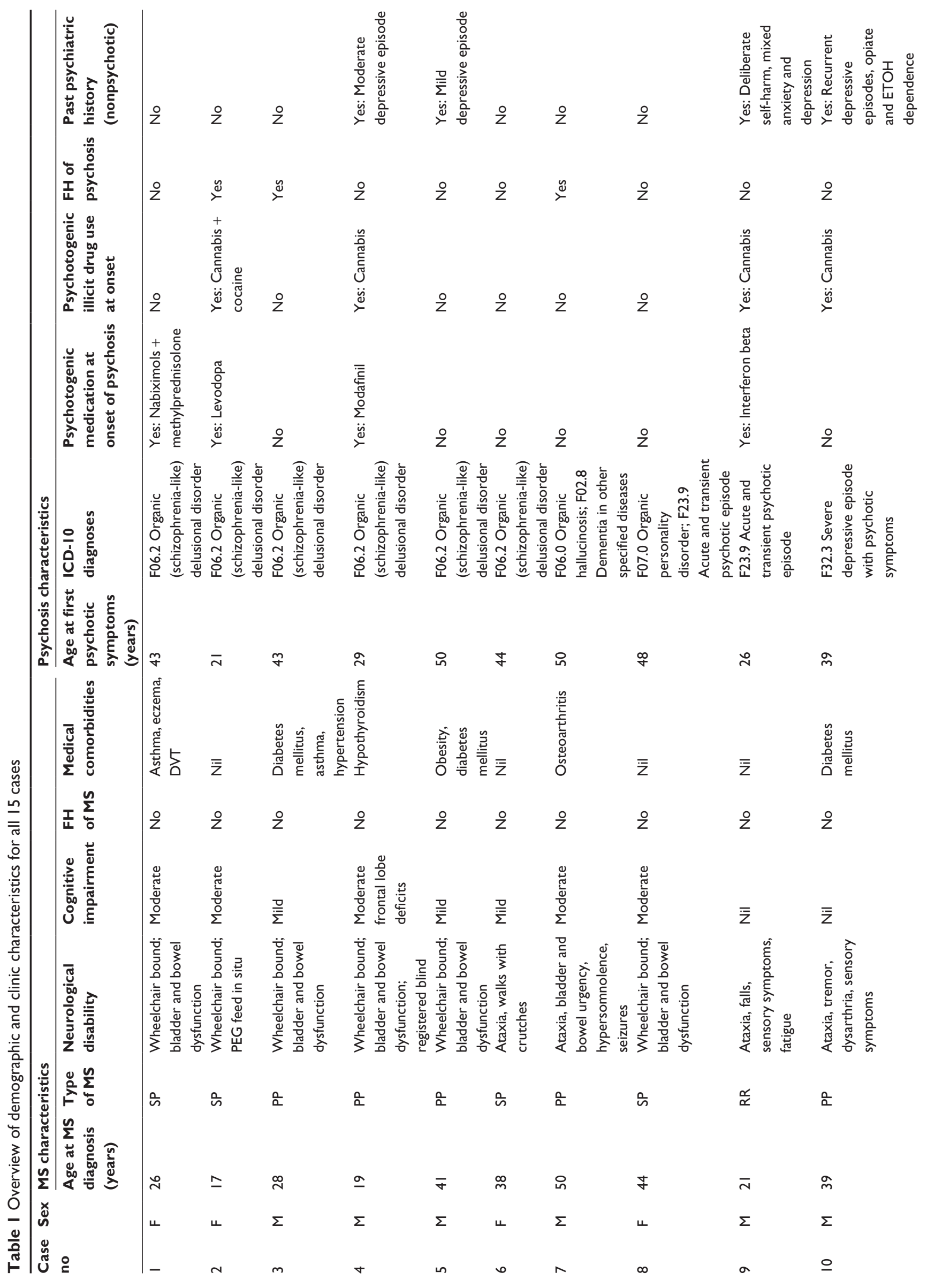




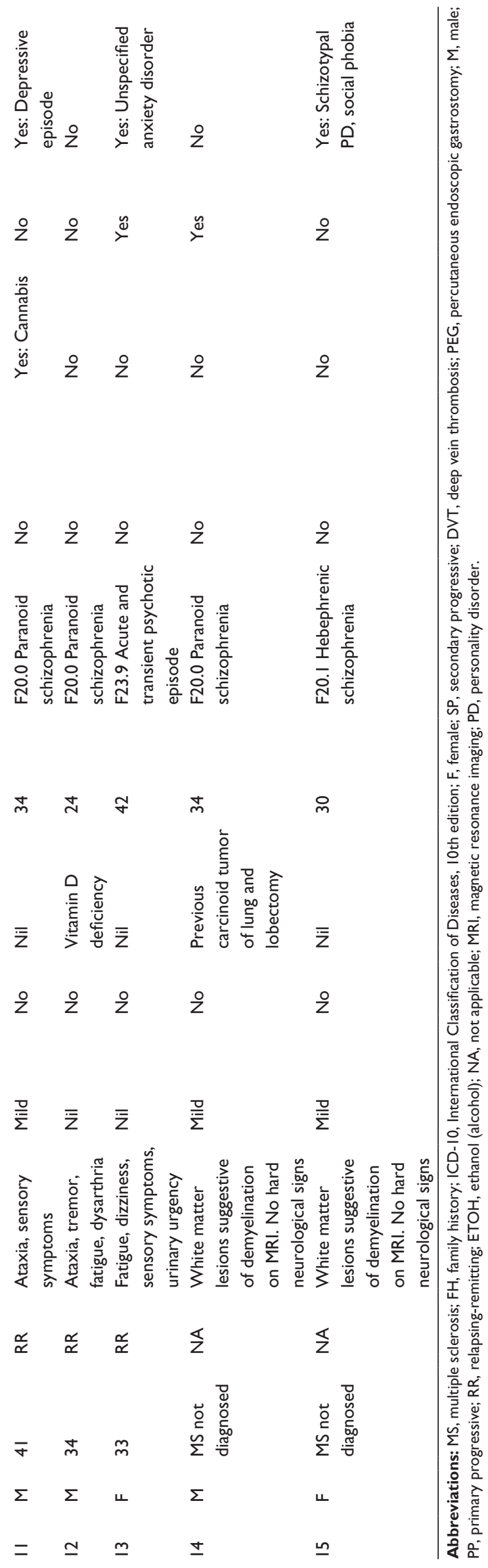

history of MS. The median age of onset of psychotic symptoms was 39 years (range, 21-50 years; mean 37 years).

Nine patients $(60 \%)$ experienced their first psychotic episode between 4 and 17 years after being diagnosed with MS. Of these, six patients were given the ICD-10 diagnosis of F06.2 Organic (schizophrenia-like) delusional disorder, and the remaining three were diagnosed with F23.9 Acute and transient psychotic episodes, one of which also had a diagnosis of F07.0 Organic personality disorder.

In three patients $(20 \%)$, first psychotic symptoms occurred a number of years (range, 7-11 years) before MS diagnosis or isolated radiological findings of demyelination, and all three had an ICD-10 diagnosis of F20.0 Paranoid schizophrenia. One such patient had a diagnosis of chronic schizophrenia for a period of 11 years before an MRI scan (performed during a psychiatric inpatient admission) showed lesions highly suggestive of demyelination in the absence of clinical neurological findings.

In the remaining three patients (20\%), onset of psychotic symptoms and MS diagnosis or MRI findings suggestive of demyelination occurred at around the same time-point, that is, within 1 year of each other. Of these, one patient presented with a predominantly cognitive syndrome and mild neurological symptoms (increased somnolence and unsteadiness of gait) and was eventually diagnosed with F02.8 Dementia in other specified diseases and F06.0 Organic hallucinosis. One patient was diagnosed with MS within months of being discharged from a psychiatric inpatient ward having been diagnosed with and treated for F32.3 Severe depressive episode with psychotic symptoms. The remaining patient, who had a history of schizotypal personality disorder and social phobia, was admitted to hospital with first-episode psychosis and during this admission had an MRI scan that revealed white matter lesions suggestive of demyelination in the absence of hard neurological signs and symptoms. During a subsequent psychiatric admission, she was diagnosed with F20.1 Hebephrenic schizophrenia.

In relation to etiological factors for psychosis in the context of MS, a third (33\%) of the patients in our sample had a positive family history of psychotic disorder in a first-degree relative and a third (33\%) were regular users of illicit drugs known to be psychotogenic, namely, cannabis. Four patients (27\%) were taking prescribed medication with established or putative psychotogenic properties at the onset of first psychotic symptoms, including steroids (methylprednisolone), levodopa, interferon beta, and oro-mucosal cannabinoid spray (nabiximols). Seven patients (47\%) had a previous history of nonpsychotic mental illness including depression, anxiety, personality disorder, and substance misuse disorder. 


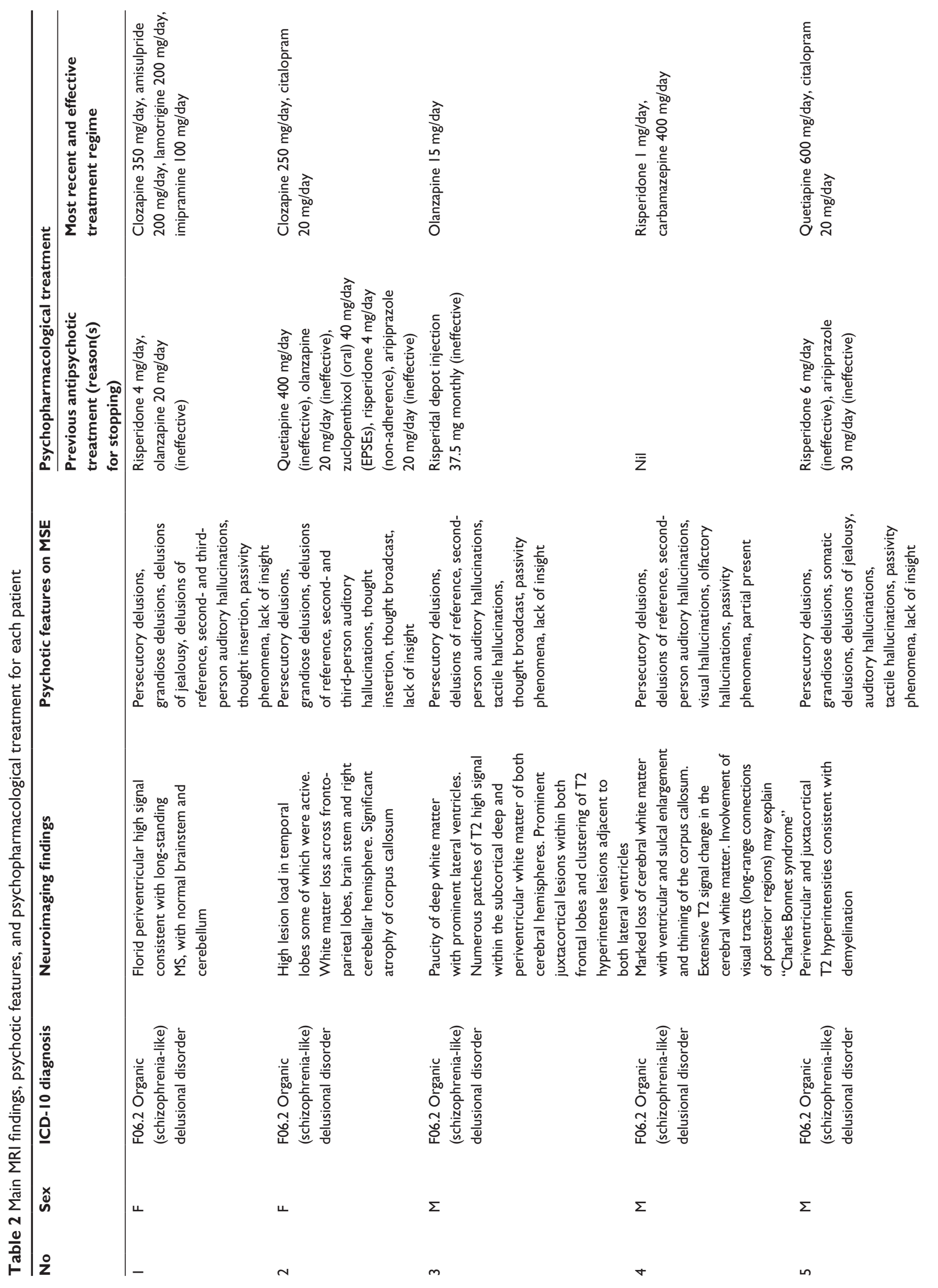



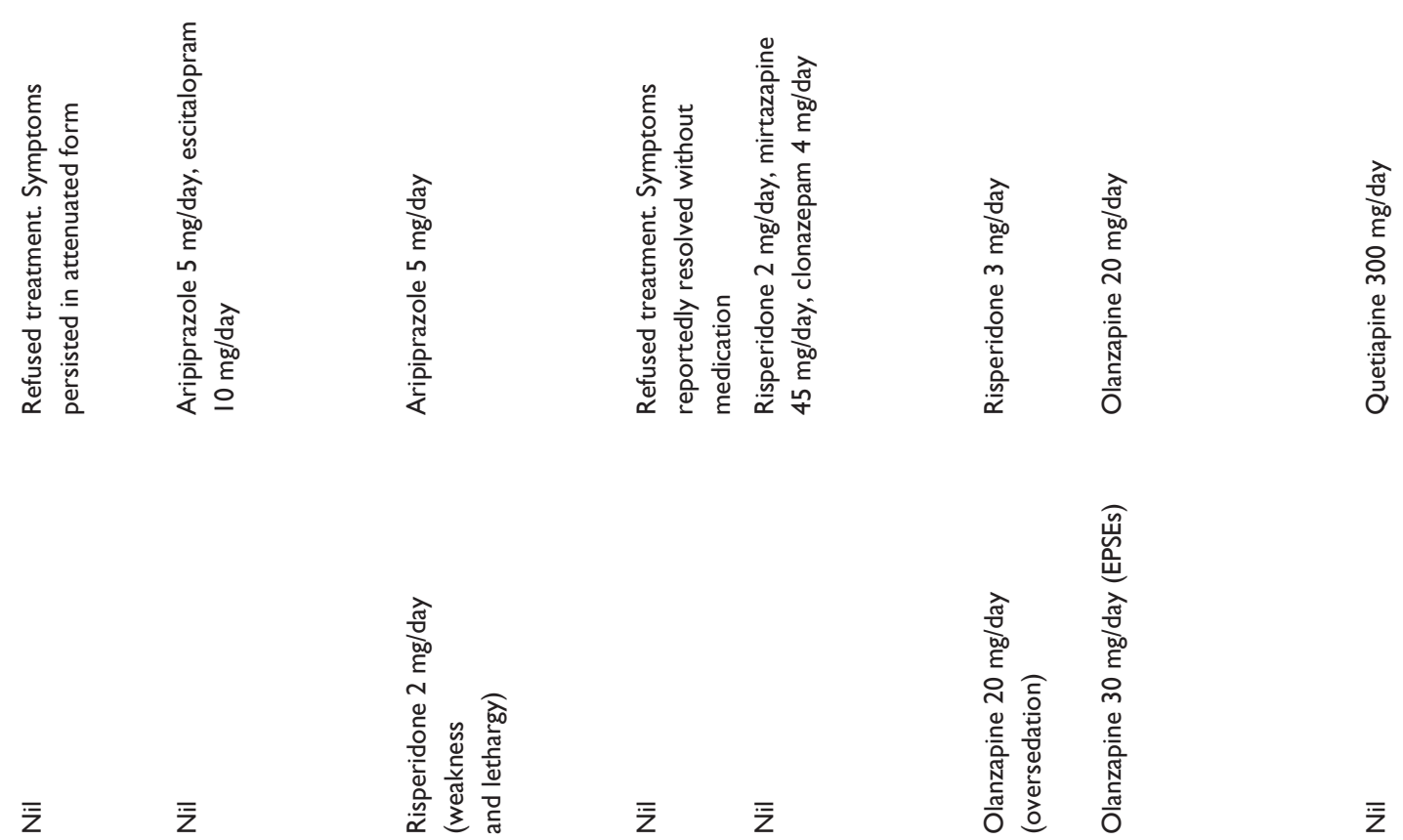

$\overline{\bar{z}}$
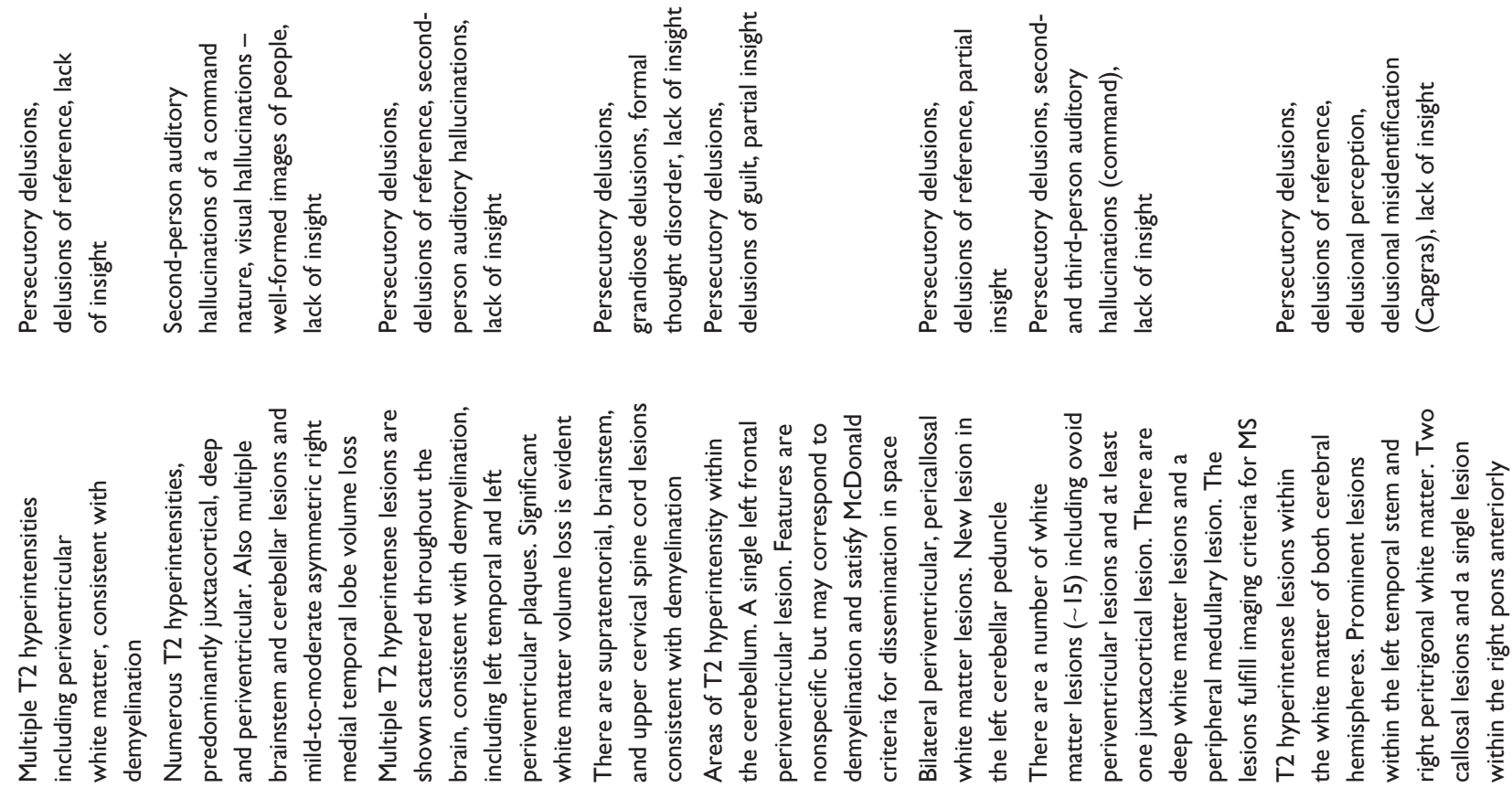

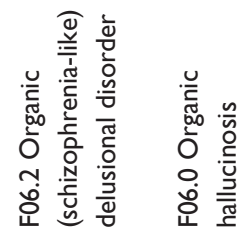

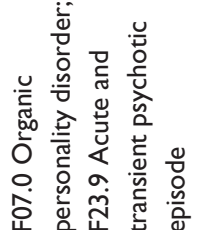
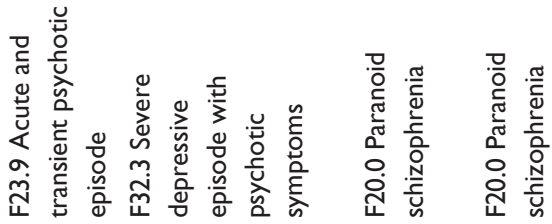

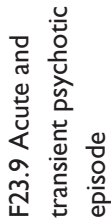

ᄂ $\quad \Sigma$

ᄂ

$\Sigma \quad \Sigma$

$\Sigma \quad \Sigma$

ᄂ 


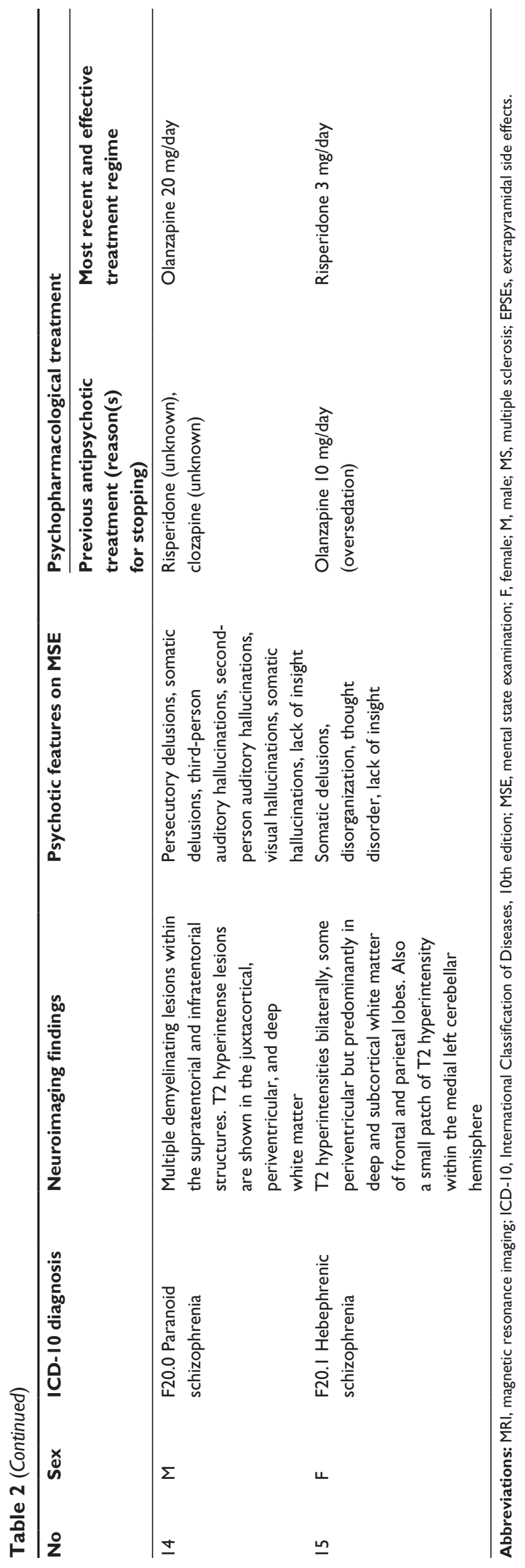

\section{Neuroimaging findings}

Thirteen of 15 cases had MRI scans that along with clinical and cerebrospinal fluid (CSF) findings confirmed a diagnosis of MS. Serial MRI scans were performed during the 5-year study period, which demonstrated dissemination of demyelinating lesions in time and space. The remaining two cases had established psychotic disorders and incidental MRI findings suggestive of demyelination in the absence of hard neurological symptoms or signs (RISs). Toward the end of the study period, Case no 14 presented to hospital with vague neurological symptoms including gait unsteadiness and was admitted but still no definitive MS diagnosis was made. Case no 15 had an initial MRI scan in 2013 during a psychiatric inpatient admission, which showed lesions suggestive of demyelination. A repeat MRI performed 1 year later showed no new lesions, and following further review of the scans by a neuroradiologist, it was concluded that the lesions were "slightly more suggestive of a vascular than an inflammatory/demyelinating etiology". Both cases are explored further in the "Discussion" section.

With regard to lesion location, 11 of 15 (73\%) MRI scans showed lesions in the periventricular white matter, which fits with the previous finding of Feinstein et al. ${ }^{14}$ However, unlike Feinstein et al, ${ }^{14}$ we did not calculate a total lesion score for each periventricular area (based on the size and number of lesions). In our study, four MRI reports (27\%) specified temporal lobe involvement, either unilateral or bilateral.

\section{Psychopathological features}

The most common psychotic symptom experienced by our sample was persecutory delusions $(13 / 15 ; 87 \%)$, followed by a lack of insight $(12 / 15 ; 80 \%)$, then delusions of reference $(8 / 15 ; 53 \%)$ and second-person auditory hallucinations $(8 / 15 ; 53 \%)$. Third-person auditory hallucinations, grandiose delusions, and passivity phenomena were experienced by $4 / 15$ (27\%); visual hallucinations, somatic hallucinations, and thought alienation phenomena occurred in three patients, while just two patients had thought disorder and one patient experienced delusional perception and delusional misidentification.

Table 3 shows the frequencies of individual psychotic features in the 15 cases.

\section{Treatment and course of psychotic disorder}

Nine patients (60\%) had been treated previously with antipsychotics that were discontinued for a variety of reasons, most commonly ineffectiveness. Two patients experienced 
Table 3 Frequencies of individual psychotic symptoms

\begin{tabular}{ll}
\hline Psychotic symptoms & $\begin{array}{l}\text { Number } \\
\text { of patients } \\
(\mathbf{N}=\mathbf{1 5}), \mathbf{n}(\%)\end{array}$ \\
\hline Persecutory delusions & $13(87)$ \\
Lack of insight & $12(80)$ \\
Delusions of reference & $8(53)$ \\
Second-person auditory hallucinations & $8(53)$ \\
Third-person auditory hallucinations & $4(27)$ \\
Grandiose delusions & $4(27)$ \\
Passivity phenomena & $4(27)$ \\
Visual hallucinations & $3(20)$ \\
Somatic hallucinations & $3(20)$ \\
Thought alienation (broadcast, insertion, withdrawal) & $3(20)$ \\
\hline
\end{tabular}

extrapyramidal side effects and three complained of feeling oversedated.

Two patients were offered antipsychotic treatment and refused it. They were deemed to have capacity to make this decision and did not meet criteria for detention in hospital under the Mental Health Act (MHA).

Most recent and most effective antipsychotic treatment in all cases was with atypical agents. Risperidone was used in four patients (27\%), olanzapine in three patients (20\%), quetiapine in two patients $(13 \%)$, and aripiprazole in two patients $(13 \%)$. The remaining two patients $(13 \%)$ were considered to be "treatment refractory" and were treated with clozapine. Case no 1 was prescribed clozapine augmentation medication, namely, amisulpride and lamotrigine, since clozapine alone did not adequately attenuate her symptoms.

Eight patients $(53 \%)$ required psychiatric inpatient admission; six of these admissions were compulsory under the MHA and two were voluntary. Four patients (27\%) were admitted to a specialist neuropsychiatry inpatient unit for integrated mental and physical health care. The other four admissions were to general acute psychiatric wards with outreach visits from the consultant neuropsychiatrist.

One-third of cases (five patients) achieved full remission of psychotic symptoms within 6 months, while two-thirds (10 patients) seemed to follow a more chronic course with ongoing attenuated psychotic symptoms while on medication.

Table 4 presents a summary of the main demographic, clinical, neuroimaging, and treatment findings.

\section{Discussion}

Our study findings show that a spectrum of psychotic disorders exists in patients with MS and that at least three clinically distinct subgroups can be delineated on the basis of the temporal relationship between the two conditions. The first and largest group consists of patients with a diagnosis of MS that predates psychosis by 4 or more years and with no previous history of psychosis. The second group consists of patients with preexisting psychotic disorders who go on to develop MS several years later. A third group consists of patients who present with symptoms of MS and psychosis

Table 4 Summary of demographic, clinical, neuroimaging, and treatment findings

\begin{tabular}{|c|c|}
\hline Sample characteristics & $\begin{array}{l}\text { Number of patients } \\
(\mathbf{N}=\mid 5)\end{array}$ \\
\hline Male & $9(60)$ \\
\hline Confirmed diagnosis of MS & $13(87)$ \\
\hline Incidental white matter lesions suggestive of demyelination on MRI (MS not confirmed) & $2(13)$ \\
\hline Age at MS onset, ${ }^{\mathrm{a}}$ years & $33(17-50)$ \\
\hline Age at psychosis onset, ${ }^{b}$ years & $39(21-50)$ \\
\hline MS diagnosis preceding first psychotic symptoms by $>$ I year & $9(60)$ \\
\hline First psychotic symptoms preceding MS diagnosis or incidental MRI findings by $>$ I year & $3(20)$ \\
\hline MS and psychosis presenting within I year of each other & $3(20)$ \\
\hline Progressive form of MS & $10(67)$ \\
\hline Family history of MS & $0(0)$ \\
\hline Family history of psychosis & $5(33)$ \\
\hline Medication known to induce psychosis prescribed at time of onset & $4(27)$ \\
\hline Psychotogenic illicit drug use at psychosis onset & $5(33)$ \\
\hline Past history of mental illness (non-psychotic) & $7(47)$ \\
\hline Periventricular lesions present on MRI & I I (73) \\
\hline Temporal lobe lesions present on MRI & $4(27)$ \\
\hline Treated with antipsychotic medication & $13(87)$ \\
\hline Required psychiatric inpatient admission for treatment of psychosis & $8(53)$ \\
\hline Remission of psychotic episode within 6 months & $5(33)$ \\
\hline
\end{tabular}

Notes: Data represented as median (range) or $\mathrm{n}(\%) .{ }^{\mathrm{a}}$ Thirteen patients included in this calculation, ${ }^{\mathrm{b}} \mathrm{mean}$ age at onset of psychosis $=37$ years.

Abbreviations: MS, multiple sclerosis; MRI, magnetic resonance imaging. 
at around the same time, a proportion of which present with solely psychiatric manifestations in the absence of hard neurological signs and symptoms.

In the following section, we characterize each subgroup in turn and discuss one patient from each subgroup in more detail as a case vignette.

\section{Subgroup I: Progressive MS predating the onset of first psychotic symptoms}

This group was composed of nine patients (60\%), seven of which were diagnosed with a progressive form of MS and had high levels of neurological disability. Of these, six were given the ICD-10 diagnosis of organic (schizophrenia-like) delusional disorder, characterized by a preponderance of positive psychotic features (persecutory delusions, delusions of reference, poor insight, and second- or third-person auditory hallucinations) and a relative absence of negative symptoms. Two such patients had psychotic syndromes that were refractory to standard antipsychotic medication and were given clozapine therapy. ${ }^{39,40}$

Case no 1 is a 44-year-old married White British female in receipt of disability benefits after previously being employed in the retail sector. She has a past medical history of atopic asthma and eczema and was first diagnosed with MS at the age of 26. She neither has a family history of MS or psychosis and reports never having used illicit substances or suffered previous mental illness. At the onset of first psychotic symptoms, she was aged 43 years and had progressive MS with significant neurological disability (wheelchair bound with severe bladder and bowel dysfunction). She had been treated with intravenous steroids within the preceding 6 months and had been taking nabiximols $\left(\right.$ Sativex $\left.{ }^{\circledR}\right),{ }^{41}$ an oro-mucosal cannabinoid preparation for muscular spasticity, on a regular basis for several years. The potential for high-dose steroids to induce psychotic symptoms is well-documented ${ }^{33-35}$ and the summary of product characteristics for nabiximol lists psychotic symptoms including hallucinations, paranoia, and delusional perception as uncommon $(\geq 1 / 1,000$ to $<1 / 100)$ adverse events. ${ }^{41}$

Her psychiatric presentation was characterized by prominent positive psychotic symptoms, namely, persecutory delusions (eg, neighbors stealing her property, being poisoned by doctors, her husband having multiple affairs, and being filmed by hidden cameras), grandiose delusions (eg, possessing special powers as a clairvoyant and sharing close relationships with various celebrities), and secondperson auditory hallucinations of a commanding nature as well as marked lack of insight (Figure 1).
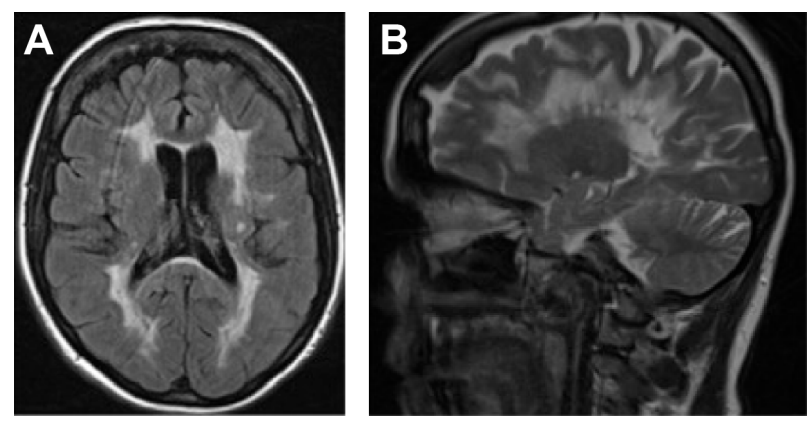

Figure I Brain MRI of Case no I.

Notes: (A) Axial FLAIR and (B) sagittal T2 images show confluent T2 hyperintensity in the periventricular cerebral white matter with mild generalized brain volume loss. Abbreviations: MRI, magnetic resonance imaging; FLAIR, fluid-attenuated inversion recovery.

Following two admissions to general adult psychiatry wards, she was transferred to a specialist neuropsychiatry unit where she was initially detained under Section 2 then Section 3 of the MHA. Her previous antipsychotic treatment consisted of risperidone $4 \mathrm{mg}$ daily followed by olanzapine $20 \mathrm{mg}$ daily. With both agents proving ineffective, clozapine therapy was commenced, slowly titrated up to $350 \mathrm{mg}$ daily and augmented with amisulpride $100 \mathrm{mg}$ twice daily. During her 9-month admission, she also exhibited marked emotional lability with intermittent periods of irritability and depressed mood. To treat her affective symptoms, lamotrigine $200 \mathrm{mg}$ daily was added and amitriptyline was switched to imipramine (100 mg daily).

Based on the putative inflammatory basis of her psychotic illness and its use as an adjunctive therapy in treatmentresistant schizophrenia, high-dose aspirin (600 mg daily) was added to her treatment regime. ${ }^{42}$ Although her psychotic symptoms persisted in attenuated form at discharge, it was felt that the aforementioned medication regime in combination with the therapeutic milieu of the neuropsychiatric unit lessened the patient's distress and the impact on her interpersonal functioning. The patient was discharged to a residential neurorehabilitation center with input from a visiting neuropsychiatrist as well as being followed up by her previous Community Mental Health Team.

\section{Subgroup 2: First psychotic symptoms predating diagnosis of MS or RIS}

The second group consists of three patients, all with an ICD-10 diagnosis of paranoid schizophrenia several years before they were diagnosed with MS (and in one case an RIS). In this group, the latency period between onset of first psychotic symptoms and either MS diagnosis or an RIS ranged from 7 to 12 years. Two of these patients had an established 
diagnosis of MS at the time they were referred for inclusion in this study. The remaining patient did not have an established MS diagnosis and was still under investigation by neurologists at the time he was referred to one of the authors (AD) for a neuropsychiatric opinion.

Case no 14 is a single, unemployed 46-year-old AfricanCaribbean male with a diagnosis of paranoid schizophrenia since the age of 34 and multiple previous psychiatric inpatient admissions, mostly involuntarily under the MHA. He has a positive family history of psychosis in his father and denies misuse of illicit substances including cannabis. His current treatment for schizophrenia is olanzapine $30 \mathrm{mg}$ daily, and he reports adhering to this consistently. He was previously trialed on clozapine medication, but this was discontinued due to suspected clozapine-induced myocarditis. He has a past medical history of right lung lobectomy for a benign carcinoid tumor and no family history of MS. His symptoms of schizophrenia over the previous 12 years have been predominantly positive, namely, auditory and visual hallucinations and bizarre somatic experiences, with a relative absence of negative symptoms.

An MRI brain scan was performed in May 2015 during a psychiatric inpatient admission because of subacute cognitive changes including confusion, disorientation, and mild deficits in short-term memory "to rule out an underlying organic cause". The scan was reported as showing "multiple T2 hyperintense lesions in keeping with demyelination" and triggered a referral to neurology. A neurologist performed clinical examination in July 2015 at which time "no focal (neurological) abnormalities" were noted. A second neurological assessment was carried out in October 2015 by a different neurologist with expertise in MS and was reported as "normal, bar slightly reduced lower leg reflexes, slightly slower finger-nose testing, and slight unsteadiness on heel-toe walking". At this stage, the diagnosis given was of "an RIS", and the plan was for a repeat MRI scan and further clinical review in 6 months to look for evidence of dissemination in time and space.

At assessment in neuropsychiatry outpatient clinic in early January 2016, Case no 14 reported experiencing a range of somatic symptoms on a daily basis since his diagnosis of schizophrenia in 2003 but was clear that these had become more intense and distressing in the previous year. During this consultation, his experiences were described as "beginning with voices" saying things such as "we' re going to pull your eyes down your body" or "we'll take it out" followed by somatic experiences lasting several minutes before spontaneously resolving. These somatic experiences include visual disturbances described as his eyes feeling "like they're fading, getting weak, light, like the substance has come out of them" or a sensation of weakness in both legs "like the nourishment is sucked out". In addition, the patient described a particularly distressing experience that had occurred 2 weeks before: "Something came out of my sides like a stabilizer. I felt like I was going out of my frame. By frame I mean body. I felt like my spirit came out. I was sinking in my frame."

In mid-January 2016 (2 months before his scheduled follow-up with neurology), Case no 14 presented to the Accident and Emergency department with a 24-hour history of worsening "unsteadiness" and "not feeling himself due to nausea, lethargy, and weak legs". At this time, a repeat MRI scan was performed that showed "a new demyelinating lesion in the right periventricular white matter, abutting the trigone". During his brief hospitalization, the same neurologist who had seen him in October 2015 reviewed him. After looking at his latest MRI scans, it was concluded that he should not be given steroids or further investigated for MS at this juncture.

At the time of writing, Case no 14 had been seen on one further occasion in outpatient clinic by the same neurologist following another attendance at A\&E with an "out-of-body, detached sensation". At this review, the patient once again described feeling "as if his life has been sucked out of his head and that he may die". The clinical impression following this review was that these unusual somatic experiences were not suggestive of demyelination and were more likely to relate to the patient's psychosis. In the absence of neurological symptoms, a diagnosis of MS was not made and a lumbar puncture was not performed. He received a diagnosis of an RIS with a medical plan to be reviewed again in neurology outpatient clinic in a further 6 months (Figure 2).

The term RIS was first used by Moore and Okuda ${ }^{43}$ to describe patients with no obvious past or present neurological symptoms indicative of MS, normal neurological examination, and evidence of white matter lesions on MRI scan that fulfill the radiological criteria for MS. The clinical and prognostic implications of such lesions in patients with RIS continue to be debated, yet existing evidence suggests that patients with RIS are at higher risk of developing MS over time with two-thirds showing radiological progression and one-third developing clinical neurological symptoms after 5 years of follow-up. ${ }^{44,45}$

A study by Lubman et $\mathrm{al}^{46}$ investigating incidental MRI abnormalities in both healthy controls and patients with psychotic disorders found an increased prevalence of incidental brain abnormalities in patients with chronic schizophrenia 

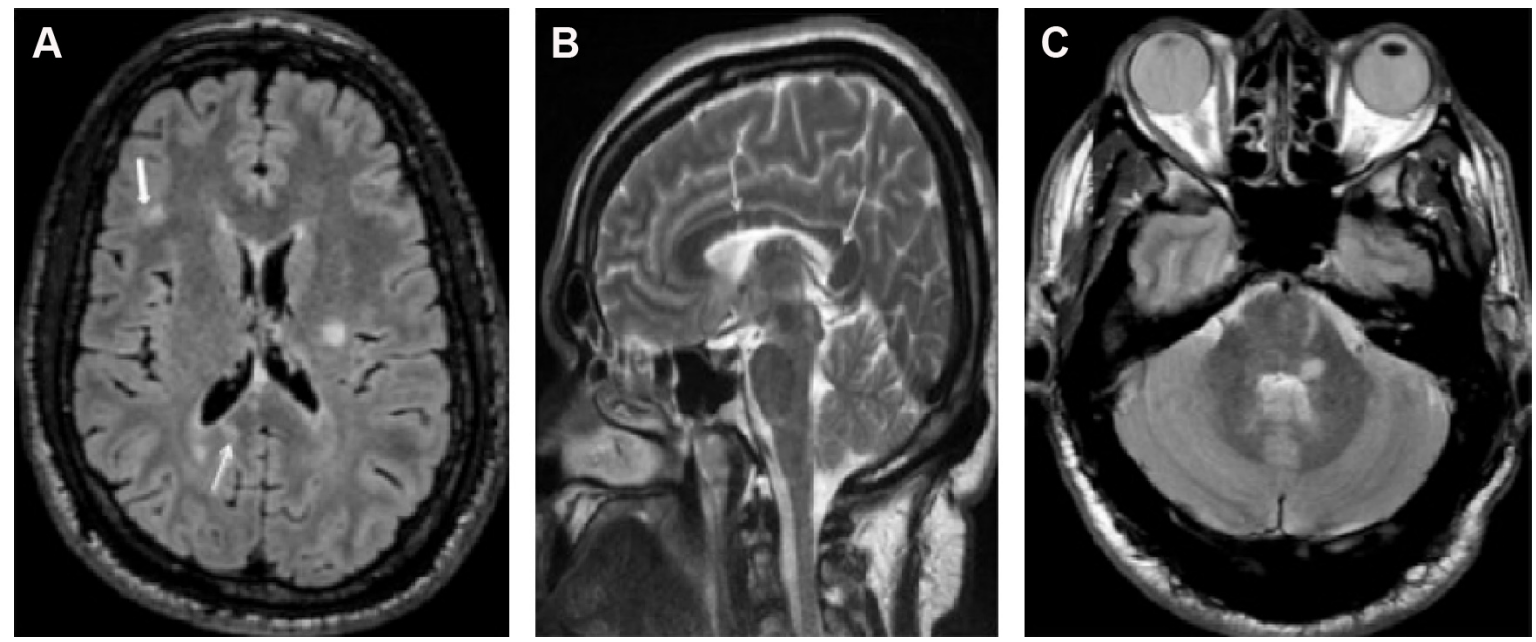

Figure 2 Brain MRI of Case no 14.

Notes: (A) Axial FLAIR image shows a low lesion load. Lesions are shown in periventricular (arrow) and juxtacortical locations (block arrow). (B) Sagittal T2 image shows lesions along the callososeptal interface (arrows). (C) Axial T2 image shows infratentorial lesions in the left hemipons.

Abbreviations: MRI, magnetic resonance imaging; FLAIR, fluid-attenuated inversion recovery.

when compared to controls and patients with first-episode psychosis. The most commonly reported abnormal findings across the study (in all three groups) were of white matter abnormalities and hippocampal abnormalities. From a total sample of 340 patients, 90 patients had chronic schizophrenia and $23.3 \%$ of these were found to have MRI white matter hyperintensities (WMHs) compared to $11.3 \%$ of healthy controls and $6.5 \%$ of patients with first-episode psychosis. While a majority of white matter abnormalities were simply reported as "WMHs", five subjects were reported to have "possible white matter demyelination", of which one was given a definitive diagnosis of MS and another who was initially extensively investigated by neurologists, and not diagnosed with MS, but then admitted to a neurology unit with optic neuritis 2 years later.

This study offers two explanations for its main finding that there is a higher prevalence of incidental brain abnormalities in patients with chronic schizophrenia. The first is that the higher incidence of brain abnormalities reflects underlying pathology related to the illness process. The second is that chronic schizophrenia patients may be less likely to report or be investigated for underlying CNS pathology such as demyelination because it is masked by their psychiatric illness. The challenges of making a definitive diagnosis of MS and the potential for diagnostic overshadowing in patients with long-standing psychotic disorders are highlighted by Case no 14. Keeping such complex patients under regular neurology follow-up (as with Case no 14) is likely to minimize unnecessary delays in confirming diagnosis and commencing appropriate treatment if and when neurological symptoms arise.
While the occurrence of psychopathological features in MS is well described, the presence of exclusively psychiatric symptoms is not generally considered to be indicative of MS and for this reason is often not further investigated. Kohler et $\mathrm{al}^{26}$ described two cases of MS presenting as chronic atypical psychosis, one in which the diagnosis of MS was confirmed at autopsy and another with MRI white matter lesions and CSF positive for oligoclonal bands.

\section{Subgroup 3: First psychotic symptoms and MS or RIS occurring within I year of each other}

The third group also consists of three patients, all of whom experienced first psychotic symptoms and were diagnosed with MS within the same 1-year period. One such patient was admitted to a psychiatric ward where he was diagnosed with a severe depressive episode with psychotic features and within months of this began to experience gait ataxia, dysarthria, and tremor. An MRI scan showed evidence of dissemination in space and along with the presence of CSF oligoclonal bands confirmed the diagnosis of MS.

One patient with a previous diagnosis of schizotypal personality disorder, autistic spectrum disorder (ASD), and social phobia was admitted involuntarily to a psychiatric ward in 2013 following a deterioration in her mental state characterized by somatic delusions, disorganized thinking and lack of insight. She was initially diagnosed with an acute and transient psychotic episode in the context of schizotypy and ASD and treated with antipsychotic medication. During the admission, she had an MRI brain scan, which showed 
"T2 hyperintensities bilaterally with some periventricular lesions but predominance within the deep and subcortical white matter". She was subsequently seen by a neurologist with expertise in MS, who found no hard neurological signs on examination. Evoked visual potentials were performed and found to be normal and she was offered but declined a lumbar puncture. The MRI findings were reviewed by a neuroradiologist who felt that on balance the white matter lesions, although nonspecific in distribution, were "slightly more suggestive of a vascular than a demyelinating etiology" based on their location. She was followed up with an MRI scan $\sim 1$ year later in 2014, which did not show any new lesions. During a further psychiatric admission, again under the MHA in November 2015, she was diagnosed with hebephrenic schizophrenia and treated with risperidone $3 \mathrm{mg}$ daily. At the time of writing, she had not developed any neurological symptoms; her MRI findings remain unexplained and essentially incidental without overt clinical implications.

Case no 7 is a 50-year-old Nigerian male who worked in IT but was forced to stop this due to excessive daytime somnolence at the end of 2013 . His only past medical history is osteoarthritis of the knee. He does not have a family history of MS and has never used illicit drugs. His initial presentation was in October 2013 to his general practitioner (GP) with the vague complaints of "difficulty walking" and "increasing fatigue". One month later, he was seen again by his GP at which time he was noted to have cognitive problems including inattention and memory deficits and was referred to a memory clinic for further assessment. By January 2014, his condition had deteriorated considerably and he had become more confused, was confabulating, and reported both visual and auditory hallucinations. As a result, he was referred to a neurologist in March 2014 at which time it was noted that he was "generally slow with nystagmus on upward-gaze" but pain secondary to osteoarthritis had limited more thorough examination. An MRI brain scan performed at this time showed "multiple WMHs of both cerebral hemispheres, predominantly in the periventricular and juxtacortical regions" as well as "severe corpus callosum involvement and thinning, moderate diffuse volume loss and mild-to-moderate asymmetric right medial temporal lobe volume loss" (Figure 3). A diagnosis of chronic demyelination was made, and MS was later confirmed by the presence of oligocloncal bands in the CSF.

In September 2014, Case no 7 was reviewed in neuropsychiatry outpatient clinic by AD and found to have a psychotic disorder characterized by a predominance of hallucinatory symptoms including visual and auditory hallucinations. He reported hearing multiple voices of a derogatory and command nature instructing him to hurt himself, for example, "walk in that river" or "use that knife" as well as seeing "white images moving across the room". He also had a prominent affective component to his presentation reporting low mood, reduced appetite, anergia and amotivation, and suicidal ideation. These symptoms apparently first emerged following significant life events, namely, losing his job and separating from his wife but had worsened considerably since the onset of his distressing psychotic symptoms. In terms of management, he was commenced on a combination of aripiprazole $5 \mathrm{mg}$ daily and escitalopram $10 \mathrm{mg}$ daily and later admitted to the specialist neuropsychiatry ward for further assessment.

On admission to the neuropsychiatry unit in October 2014, Case no 7 presented with psychomotor retardation, flattened
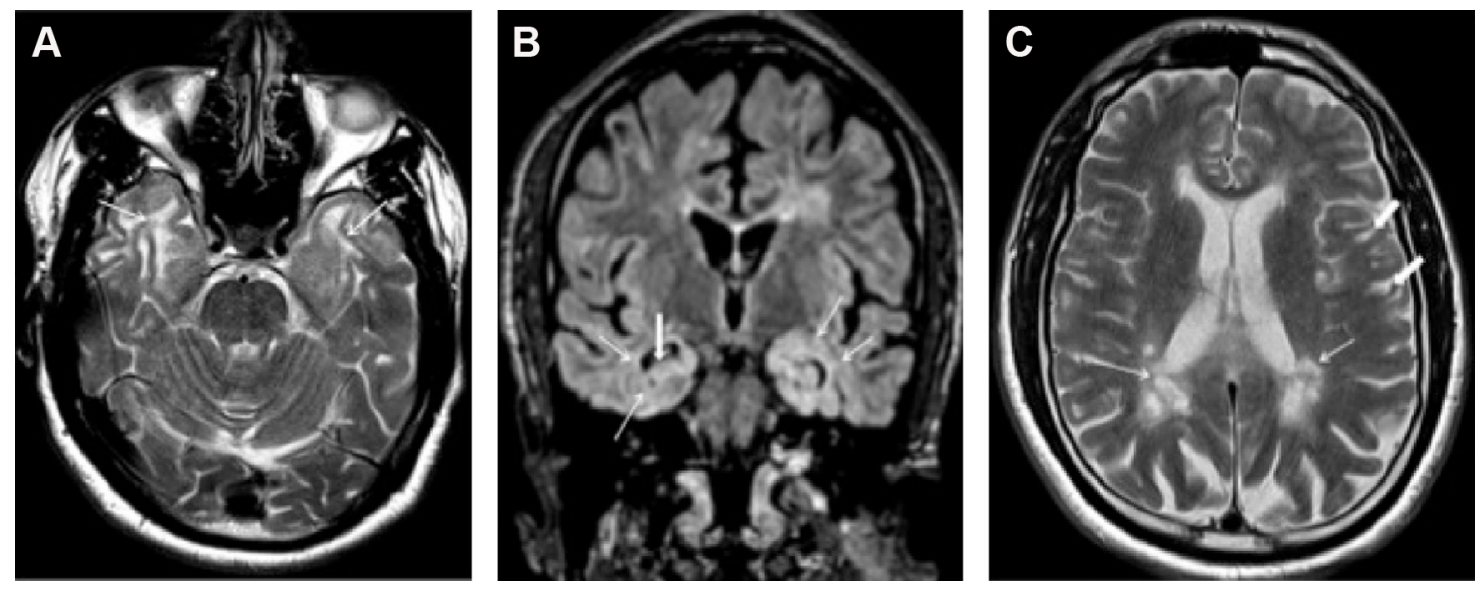

Figure 3 Brain MRI of Case no 7.

Notes: (A) Axial T2 and (B) coronal FLAIR images show confluent lesions in the anterior temporal white matter bilaterally (arrows). Some right hippocampal volume loss is also noted (block arrow). (C) Axial T2 image shows periventricular (arrows) and juxtacortical lesions (block arrows) with some volume loss.

Abbreviations: MRI, magnetic resonance imaging; FLAIR, fluid-attenuated inversion recovery. 
affect, and poverty speech as well as persistent auditory and visual hallucinations as described earlier. His walking was unsteady, and he had been prone to poor balance and falls. He also had urinary and fecal urgency with occasional incontinence. Neuropsychological evaluation revealed widespread cognitive impairment compared to premorbid levels with specific deficits in episodic memory. However, part of the assessment suggested a significant motivational component contributing to his poor test performance and likely leading to an overestimate of cognitive impairment. Physical examination revealed a "simian gait, abnormal eye movements, slowness of upper limb movements, and bilateral clonus". An electroencephalogram (EEG) was also performed during the admission that showed "frequent sharp waves and spike and wave discharges over the frontotemporal region as well as one electrical seizure originating from the right temporal region and bursts of theta activity over the frontal regions bilaterally and independently". The EEG findings were felt to be suggestive of focal epilepsy arising from the right frontal lobe. It was concluded by the neurologists that this man's MRI findings along with CSF results were indicative of PP MS with evidence of advanced demyelination.

With a short history of less severe neurological symptoms and a predominance of cognitive and psychotic symptoms at first presentation, Case no 7 fits the description of the "cognitive" or "cortical" variant of MS characterized by Assouad et $\mathrm{al}^{25}$ who refer to such cases as representing a diagnostic challenge and highlight that clinical presentation may include some or all of the following features: "1) a disease onset with cognitive or behavioral change with a subsequent severe cognitive impairment occurring early in the disease course; 2) a frequent association with other psychiatric manifestations, pseudobulbar affect, urinary incontinence, and gait disturbance suggestive of high-level gait disorder (gait apraxia); 3) infrequent visual, motor, sensory, or cerebellar abnormalities that are mild or only transiently observed during superimposed relapses; and 4) extensive white matter lesions together with rapidly progressing brain atrophy on MRI".

At onset, Case no 7 had memory loss and depressed mood that tally with the findings of a previous study that these are the more common symptoms at onset in patients with predominantly cognitive MS. Finally, the MRI findings of "severe corpus callosum thinning, moderate diffuse volume loss, and mild-to-moderate asymmetric right medial temporal lobe volume loss" found in Case no 7 correlates with the observation of severe atrophy measured as "an increase in third ventricular width compared to age-matched healthy controls" in the study of Assouad et al. ${ }^{25}$
In summary, a number of important observations can be made from our study data, some of which warrant further exploration in future research.

First, our study found a later age of onset of psychosis in MS (mean age of 37 years) compared to the general population (median age of 22 or 23 years at first presentation in patients with schizophrenia ${ }^{47,48}$ ), which is consistent with previous work by Feinstein et al, ${ }^{14}$ who found a mean age of onset of psychosis in MS of 36.6 years.

Second, in our sample, there was a preponderance of males with MS and psychotic disorders compared to females (9 males: 6 females). This fits with a previous finding by Davison et $\mathrm{al}^{49}$ who found 21 of 39 cases of psychosis in MS to be male. Interestingly, however, this gender difference is at odds with the 2:1 female-to-male ratio found in MS and the roughly equal gender rates for schizophrenia in the general population. ${ }^{50}$ It is worth noting that the large Canadian study by Patten et $\mathrm{al}^{22}$ did not find a gender difference in co-occurring MS and psychosis.

Third, our sample has a high percentage (73\%) of patients with cognitive impairment (rated as either mild or moderate but not severe). All patients in the study assessed as having "moderate" cognitive impairment had a progressive form of MS and a secondary (organic) psychotic disorder. These findings are in keeping with previous evidence of high levels of cognitive impairment in MS and the more specific finding of Borghi et $\mathrm{al}^{12,13}$ that patients with progressive MS and neuropsychiatric comorbidity such as depression are likely to have lower cognitive assessment scores.

Lastly, in the sample, the majority of patients had neurological symptoms several years before the onset of psychosis $(60 \%)$, and in this subgroup, it was more likely that 1) the patient had a progressive form of MS with high levels of neurological disability; 2) the patient's ICD-10 diagnosis was organic schizophrenia-like disorder (characterized exclusively by positive psychotic symptoms); 3 ) the patient had cognitive impairment; and 4) the MRI showed evidence of significant brain demyelination using terms such as "florid", "extensive", and "numerous" to describe hyperintense lesions most often in periventricular areas. Furthermore, most patients in this subgroup had at least one premorbid vulnerability factor (family history, psychotogenic drug use, and premorbid psychiatric history) for developing psychosis. Such a group has been described in previous studies and adds further support to the hypothesis that demyelination superimposed on premorbid vulnerability factors is responsible for the association of MS and psychosis. ${ }^{7,8}$

Of particular interest in this study are the outlying cases where psychosis predates the onset of MS or an 
RIS by several years and the demyelination hypothesis is less obviously applicable. Such cases (nos 11, 12, 14) raise the possibility of an alternative explanation for the association of MS and psychosis such as shared pathogenesis. For example, exposure to a virus at a critical developmental stage (in utero, at birth, or during childhood) as per the neurodevelopmental model of schizophrenia. ${ }^{51}$ One of the authors $(\mathrm{KO} \mathrm{C})$ of our study has also written a review article about immune factors in schizophrenia. ${ }^{52}$ The two patients in this study with RISs continue to be followed up in neuropsychiatry and neurology outpatient clinics.

\section{Limitations}

Our study has a number of limitations, not least its small sample size, and would benefit from further large-scale epidemiological studies looking at the prevalence of psychosis in the context of MS along with focused neurobiological research investigating the putative inflammatory and immunological etiology of psychosis. First, as a retrospective case series, this study made use of the results of previously performed cognitive assessments and therefore did not use the same cognitive assessment method for each patient. It follows that this study would have benefited from having all patients assessed using the same assessment tool, for example, the BRB, which is a validated assessment tool with good sensitivity as a measure of cognitive dysfunction in MS. ${ }^{53}$ Second, this study may have benefited from further analysis of education level, as in other studies of neuropsychiatric disorders in MS since this may have revealed itself to be an important risk/protective factor for psychosis in MS. Finally, we concede that the neuroimaging analysis was basic and descriptive. Future research might aim to add to the work done by Feinstein et $\mathrm{a}^{14}$ by using more detailed volumetric neuroimaging methods such as voxel-based morphometry to better elucidate any differences between the brains of MS patients with and without psychosis.

\section{Conclusion}

Psychosis in MS is a relatively uncommon neuropsychiatric presentation when compared to the burden of affective disorders and cognitive impairment in MS. Nevertheless, it has a significant additional impact on social and occupational functioning and quality of life. Furthermore, untreated psychosis can adversely affect a patients' adherence to their MS treatment regime and thus give rise to worse neurological outcomes.

Our study further characterizes the spectrum of psychotic disorders in MS and in doing so highlights some of the particular diagnostic and therapeutic challenges that such disorders can pose for neurologists and psychiatrists alike. By describing the relative efficacy and common side effects of the psychopharmacological medication regimes used in our patients, we provide examples of potential interventions for this complex patient population. Further antipsychotic trials in patients with psychosis in MS are required to advance existing descriptive studies and establish a more robust evidence base to guide treatment choices.

\section{Disclosure}

The authors report no conflicts of interest in this work.

\section{References}

1. National Institute of Neurological Disorders and Stroke (NINDS) [homepage on the Internet]. NINDS Multiple Sclerosis Information Page; 2015. Available from: www.ninds.nih.gov. Accessed March 6, 2016.

2. Leary S, Giovannoni G, Howard R, et al. Multiple sclerosis and demyelinating diseases. In: Clarke C, Howard R, Rossor M, Shorvon S, editors. Neurology: A Queen Square Textbook. Oxford: Wiley-Blackwell; 2009:411-448.

3. MS Society. MS in the UK January 2016 (PDF). Available from: www. mssociety.org.uk/ms-resources/ms-uk. Accessed October 11, 2016.

4. Mackenzie IS, Morant SV, Bloomfield GA, MacDonald TM, O'Riordan J. Incidence and prevalence of multiple sclerosis in the UK 1990-2010: a descriptive study in the General Practice Research Database. J Neurol Neurosurg Psychiatry. 2014;85(1):76-84.

5. World Health Organization. Atlas: Multiple Sclerosis Resources in the World 2008 (PDF). Geneva: World Health Organization; 2008:5-16. Accessed October 11, 2016.

6. Sibley W. Diagnosis and course in multiple sclerosis. In: Rao S, editor. Neurobehavioural Aspects of Multiple Sclerosis. New York: Oxford University Press; 1990:5-14.

7. Feinstein A. The neuropsychiatry of multiple sclerosis. Can J Psychiatry. 2004;49(3):57-63.

8. Ghaffar O, Feinstein A. The neuropsychiatry of multiple sclerosis: a review of recent developments. Curr Opin Psychiatry. 2007;20(3):278-285.

9. Paparrigopoulos T, Ferentinos P, Kouzoupis A, Koutsis G, Papadimitriou GN. The neuropsychiatry of multiple sclerosis: focus on disorders of mood, affect and behaviour. Int Rev Psychiatry. 2010;22(1): $14-21$.

10. Diaz-Olavarrieta C, Cummings JL, Velazquez J, Garcia de la Cadena C. Neuropsychiatric manifestations of multiple sclerosis. J Neuropsychiatry Clin Neurosci. 1999;11(1):51-57.

11. Rao SM, Leo GJ, Bernardin L, Unverzagt F. Cognitive dysfunction in multiple sclerosis. I. Frequency, patterns, and prediction. Neurology. 1991; 41(5):685-691.

12. Borghi M, Cavallo M, Carletto S, et al. Presence and significant determinants of cognitive impairment in a large sample of patients with multiple sclerosis. PLoS One. 2013;8(7):e69820.

13. Borghi M, Carletto S, Ostacoli L, et al. Decline of neuropsychological abilities in a large sample of patients with multiple sclerosis: a two-year longitudinal study. Front Hum Neurosci. 2016;10:282.

14. Feinstein A, Du Bourlay G, Ron M. Psychotic illness in multiple sclerosis. A clinical and magnetic resonance imaging study. Br J Psychiatry. 1992; 161:680-685.

15. Chwastiak L, Ehde DM, Gibbons LE, Sullivan M, Bowen JD, Kraft GH. Depressive symptoms and severity of illness in multiple sclerosis: epidemiologic study of a large community sample. Am J Psychiatry. 2002;159(11):1862-1868.

16. Mohr DC, Goodkin DE, Likosky W, Gatto N, Baumann KA, Rudick RA. Treatment of depression improves adherence to interferon beta- $1 \mathrm{~b}$ therapy for multiple sclerosis. Arch Neurol. 1997;54(5):531-533. 
17. Tsivgoulis G, Triantafyllou N, Papageorgiou C, et al. Associations of the Expanded Disability Status Scale with anxiety and depression in multiple sclerosis outpatients. Acta Neruol Scand. 2007;115(1):67-72.

18. Amato MP, Ponziani G, Rossi F, Liedl CL, Stefanile C, Rossi L. Quality of life in multiple sclerosis: the impact of depression, fatigue and disability. Mult Scler. 2001;7(5):340-344.

19. Berrigan LI, Fisk JD, Patten SB, et al; CIHR Team in the Epidemiology and Impact of Comorbidity on Multiple Sclerosis (ECoMS). Healthrelated quality of life in multiple sclerosis: direct and indirect effects of comorbidity. Neurology. Epub 2016 Mar 9.

20. Ostacoli L, Carletto S, Borghi M, et al. Prevalence and significant determinants of post-traumatic stress disorder in a large sample of patients with multiple sclerosis. J Clin Psychol Med Settings. 2013; 20(2):240-246.

21. Kosmidis MH, Giannakou M, Messinis L, Papathanasopoulos P. Psychotic features associated with multiple sclerosis. Int Rev Psychiatry. 2010;22(1):55-66.

22. Patten SB, Svenson LW, Metz LM. Psychotic disorders in MS: population-based evidence of an association. Neurology. 2005;65(7): 1123-1125.

23. Pine DS, Douglas CJ, Charles E, Davies M, Kahn D. Patients with multiple sclerosis presenting to psychiatric hospitals. J Clin Psychiatry. 1995;56(7):297-306.

24. Lyoo IK, Seol HY, Byun HS, Renshaw PF. Unsuspected multiple sclerosis in patients with psychiatric disorders: a magnetic resonance imaging study. J Neuropsychiatry Clin Neurosci. 1996;8(1):54-59.

25. Assouad R, Louapre C, Tourbah A, et al. Clinical and MRI characterization of MS patients with a pure and severe cognitive onset. Clin Neurol Neurosurg. 2014;126:55-63.

26. Kohler J, Heilmeyer H, Volk B. Multiple sclerosis presenting as chronic atypical psychosis. J Neurol Neurosurg Psychiatry. 1988;51(2): 281-284.

27. Clarke T, Wadhwa U, Leroi I. Psychotic depression. An atypical initial presentation of multiple sclerosis. Psychosomatics. 1998;39(1):72-75.

28. Yadav R, Zigmond AS. Temporal lobe lesions and psychosis in multiple sclerosis. BMJ Case Rep. 2010;2010:bcr0120102651.

29. Engler F, Vetter P. Affektive und schizophrene syndromen bei multipler sklerose. Überprüfung der literatur und fallberichte. [Affective and schizophrenic syndromes in multiple sclerosis. Review of the literature and case reports]. Schweiz Arch Neurol Psychiatr. 1991;142(4):367-378. German.

30. Drake ME Jr. Acute paranoid psychosis in multiple sclerosis. Psychosomatics. 1984;25(1):60-61, 65.

31. Dieterle L, Staudacher T. [Schizophreniform psychosis with polydipsia and electrolyte imbalance in multiple sclerosis]. Nervenarzt. 1998;69(8): 698-701. German.

32. Reiss JP, Sam D, Sareen J. Psychosis in multiple sclerosis associated with left temporal lobe lesions on serial MRI scans. J Clin Neurosci. 2006;13(2):282-284.

33. Lewis DA, Smith RE. Steroid-induced psychiatric syndromes. A report of 14 cases and a review of the literature. J Affect Disord. 1983; 5(4):319-332.

34. Sirois F. Steroid psychosis: a review. Gen Hosp Psychiatry. 2003;25(1): 27-33.

35. Sechi GP, Piras MR, Demurtas A, Tanca S, Rosati G. Dexamethasoneinduced schizoaffective-like state in multiple sclerosis: prophylaxis and treatment with carbamazepine. Clin Neuropharmacol. 1987;10(5): $453-457$.

Neuropsychiatric Disease and Treatment

\section{Publish your work in this journal}

Neuropsychiatric Disease and Treatment is an international, peerreviewed journal of clinical therapeutics and pharmacology focusing on concise rapid reporting of clinical or pre-clinical studies on a range of neuropsychiatric and neurological disorders. This journal is indexed on PubMed Central, the 'PsycINFO' database and CAS,
36. Goeb JL, Even C, Nicolas G, Gohier B, Dubas F, Garré JB. Psychiatric side effects of interferon-beta in multiple sclerosis. Eur Psychiatry. 2006; 21(3):186-193.

37. McDonald WI, Compston A, Edan G, et al. Recommended diagnostic criteria for multiple sclerosis: guidelines from the International Panel on the diagnosis of multiple sclerosis. Ann Neurol. 2001;50(1):121-127.

38. Polman CH, Reingold SC, Banwell B, et al. Diagnostic criteria for multiple sclerosis: 2010 revisions to the McDonald criteria. Ann Neurol. 2011;69(2):292-302.

39. Safferman AZ, Kane JM, Aronowitz JS, Gordon MF, Pollack S, Lieberman JA. The use of clozapine in neurologic disorders. $J$ Clin Psychiatry. 1994;55(suppl B):98-101.

40. Chong SA, Ko SM. Clozapine treatment of psychosis associated with multiple sclerosis. Can J Psychiatry. 1997;42(1):90-91.

41. Electronic Medicines Compendium (eMC) [webpage on the Internet]. Sativex Oromucosal Spray - Summary of Product Characteristics (SPC) - (eMC). Available from: www.medicines.org.uk/emc/ medicine/23262. Accessed October 11, 2016.

42. Nitta M, Kishimoto T, Müller N, et al. Adjunctive use of nonsteroidal anti-inflammatory drugs for schizophrenia: a meta-analytic investigation of randomized controlled trials. Schizophr Bull. 2013;39(6): 1230-1241.

43. Moore F, Okuda DT. Incidental MRI anomalies suggestive of multiple sclerosis: the radiologically isolated syndrome. Neurology. 2009; 73(20): 1714.

44. Granberg T, Martola J, Kristoffersen-Wiberg M, Aspelin P, Fredrikson S. Radiologically isolated syndrome - incidental magnetic resonance imaging findings suggestive of multiple sclerosis, a systematic review. Mult Scler. 2013;19(3):271-280.

45. Okuda DT, Siva A, Kantarci O, et al. Radiologically isolated syndrome: 5-year risk for an initial clinical event. PLoS One. 2014;9(3):e90509.

46. Lubman DI, Velakoulis D, McGorry PD, et al. Incidental radiological findings on brain magnetic resonance imaging in firstepisode psychosis and chronic schizophrenia. Acta Psychiatr Scand. 2002;106(5):331-336.

47. Jones P, Rodgers B, Murray R, Marmot M. Child development risk factors for adult schizophrenia in the British 1946 birth cohort. Lancet. 1994;344(8934):1398-1402.

48. Lauronen E, Miettunen J, Veijola J, Karhu M, Jones PB, Isohanni M. Outcome and its predictors in schizophrenia within the Northern Finland 1966 Birth Cohort. Eur Psychiatry. 2007;22(2):129-136.

49. Davison, K, Bagley, CR. Schizophrenia-like psychoses associated with organic disorders of the central nervous system: a review of the literature. Current Problems in Neuropsychiatry: Schizophrenia, Epilepsy, the Temporal Lobe (ed. R. N. Herrington). 1969. 113-184.

50. Abel KM, Drake R, Goldstein JM. Sex differences in schizophrenia. Int Rev Psychiatry. 2010;22(5):417-428.

51. Fatemi HS, Folsom TD. The neurodevelopmental hypothesis of schizophrenia, revisited. Schizophr Bull. 2009;35(3):528-548.

52. Dev KK, O'Connell KE. The immune and metabolic factors of schizophrenia. J J Psych Behav Sci. 2014;1(1):004.

53. Boringa JB, Lazeron RH, Reuling IE. The brief repeatable battery of neuropsychological tests: normative values allow application in multiple sclerosis clinical practice. Mult Scler. 2001;7(4):263-267.

and is the official journal of The International Neuropsychiatric Association (INA). The manuscript management system is completely online and includes a very quick and fair peer-review system, which is all easy to use. Visit http://www.dovepress.com/testimonials.php to read real quotes from published authors. 